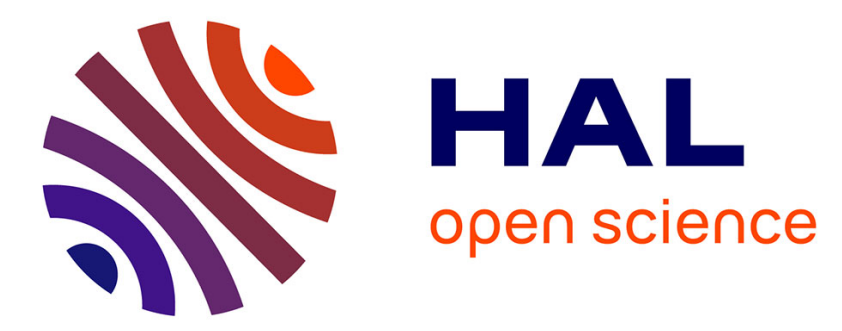

\title{
Long-term optical transmittance measurements of silica nanofibers
}

\author{
Maha Bouhadida, Philippe Delaye, Sylvie Lebrun
}

\section{To cite this version:}

Maha Bouhadida, Philippe Delaye, Sylvie Lebrun. Long-term optical transmittance measurements of silica nanofibers. Optics Communications, 2021, 500, pp.127336. 10.1016/j.optcom.2021.127336 . hal-03357339

\section{HAL Id: hal-03357339 \\ https://hal-iogs.archives-ouvertes.fr/hal-03357339}

Submitted on 30 Sep 2021

HAL is a multi-disciplinary open access archive for the deposit and dissemination of scientific research documents, whether they are published or not. The documents may come from teaching and research institutions in France or abroad, or from public or private research centers.
L'archive ouverte pluridisciplinaire HAL, est destinée au dépôt et à la diffusion de documents scientifiques de niveau recherche, publiés ou non, émanant des établissements d'enseignement et de recherche français ou étrangers, des laboratoires publics ou privés. 


\title{
Long-Term Optical Transmittance Measurements of Silica Nanofibers
}

Maha Bouhadida, Philippe Delaye, Sylvie Lebrun

Université Paris-Saclay, Institut d'Optique Graduate School, CNRS, Laboratoire Charles Fabry, 91127, Palaiseau, France.

Contact: sylvie.lebrun@institutoptique.fr +33 (1) 64533457

\begin{abstract}
:
Optical nanofibers are highly attractive tools that are used in an increasing number of applications. However, at these radii typically smaller than the wavelength, the optical field is highly sensitive to dusts that rapidly degrade the optical transmittance, which is an issue for their use and storage. In this work we propose and characterize simple and efficient solutions. The chosen nanofiber radius is $500 \mathrm{~nm}$. Firstly, we make systematic and long-term measurements (over several months) of nanofiber optical transmittance at $1.5 \mu \mathrm{m}$ in two protected environments that can be easily found in almost any laboratory and differing by their levels of cleanliness. We show that the rate of decrease of the optical transmittance is very low when the nanofiber is put in a simple protection box and ten times lower when the nanofiber is put in an airtight box. Secondly, the repetition of these measurements gives a mean value of this rate of $5.2 \times 10^{-2} \% /$ day in the protection box and $-6.3 \times 10^{-3} \% /$ day in the airtight box. Thirdly, we perform the optical transmittance measurements on two nanofiber lengths $(2 \mathrm{~cm}$ and $7 \mathrm{~cm}$ ) and show that there is no significant difference on the rate of degradation between the two lengths. These results also enable to predict the value of the optical transmittances after several months or even years, which is a key parameter for most of the applications using nanofibers.
\end{abstract}

Keywords: silica nanofibers, optical transmittance, evanescent field, applications of nanofibers, guided optics at the nanoscale

\section{Introduction}

Optical silica nanofibers are fabricated by pulling fibers until reaching diameters comparable or smaller than the light wavelength. The nanofiber is linked to the untapered end-fibers via two conical transition sections named the tapers (see Figure 1). In the tapers, the volume of the optical guided mode is progressively reduced and the intensity in the silica as well as in the evanescent field are strongly increased. 
Optical power transfer in the nanofiber can be higher than 99\% [1] and the nanofiber can be easily inserted in fibered networks with very low losses. Thanks to the strong evanescent field around the ultrathin waist region, nanofibers are powerful and versatile tools for many applications such as optical manipulation [2,3], sensing [4], nonlinear optics $[5,6,7,8]$, microcavities [9], atomic physics [10,11], and different studies of light-matter interactions in both classical and quantum regimes [12]. The strong confinement of the light inside the nanofiber itself has also enabled the generation of efficient nonlinear effects, such as the realization of supercontinuum [13].

Despite all these attractive applications, the nanofiber performances are limited by the degradation of its optical transmittance during time. Most of the time, nanofibers are fabricated in clean rooms to avoid deposition of dusts that can damage them during the pulling process. Once fabricated, the nanofibers are put on an experimental setup which is often out of the clean room for practical reasons. However, as it is usually observed by experimenters, the optical transmittance of a nanofiber placed in a standard laboratory environment rapidly falls to zero. It has been previously demonstrated that this degradation is mostly due to the dust particles deposited on its surface [14]. These particles accumulate and scatter the guided light, leading to the decrease of the transmittance [15]. Embedding nanofibers in polymers has been proposed as a protection [16]. However, apart from being complex, it may be not useable for some applications that for example use interaction of the evanescent field with the surrounding medium. So, it is important to have a simple and efficient solution for experiments that need long-time acquisitions and also to store nanofibers once fabricated, in particular to gain time for repetitive experiments.

In this work, we perform systematic and long-term measurements (over several months) of nanofiber optical transmittance in two protected environments that can be easily found in almost any laboratory and differing by their levels of cleanliness. We show that the nanofiber optical transmittance degradation is very low in both environment we chose and that an additional degree of cleanliness enables to slow down the degradation. We have also tested the influence of the nanofiber length. We have observed that this length has no impact on the degradation, which is an interesting point for applications that need long nanofibers, for example in nonlinear optics. Our results also enable to predict the value of the transmittance over very large duration which is an important parameter to control for most applications based on nanofibers. 
The outline of this paper is the following: we firstly present a theoretical analysis of the guided mode in a nanofiber to show the importance of the evanescent field probing the dust particles at the surface of the nanofiber and its tapers. Then we present the experimental setup and the different measurements we have made. Finally, we show and discuss the experimental results.

\section{Analysis of the optical guided mode}

The nanofiber geometry is presented in Figure 1. In the nanofiber part, the silica core is fully melted in the silica cladding of the initial fiber and the optical field is guided by the interface silica/air. Due to the strong confinement of light in the nanofiber and the high index contrast between silica $\left(n_{s i}=1.45\right)$ and air $\left(n_{\text {air }}=1\right)$, a vectorial mode analysis is used to describe the mode propagation [17].

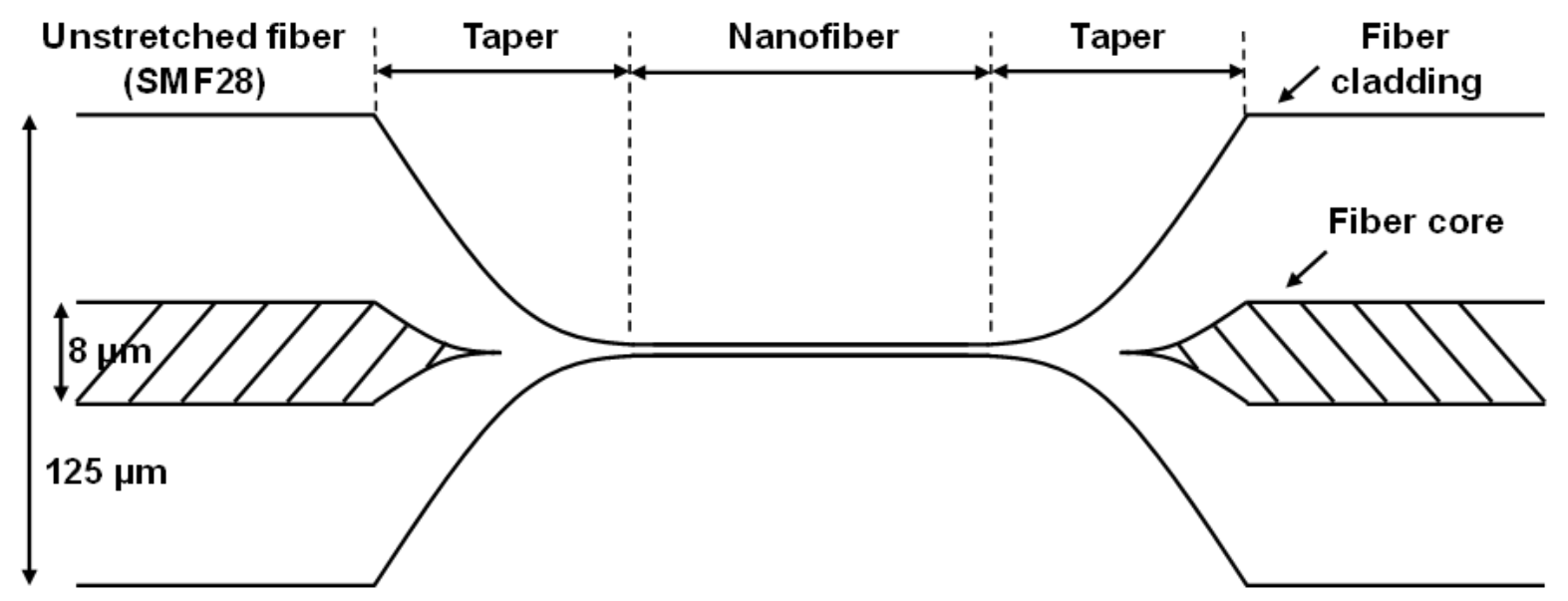

Figure 1. Geometry of a nanofiber and its tapers.

Figure 2 (top) shows the z-component of the Poynting vector of the fundamental mode HE11 at $1.5 \mu \mathrm{m}$ for a nanofiber radius of $500 \mathrm{~nm}$ defined by $S_{z}=(\vec{e} \times \vec{h}) \cdot \vec{z} \cdot \vec{e}$ is the electric field, $\vec{h}$ is the magnetic field and $\vec{z}$ is the direction of propagation. The mode exhibits a strong evanescent part, explaining its high sensitivity to dust particles that are electrostatically attracted at its surface. Figure 2 (bottom) shows the fraction of the evanescent field versus the radius of the nanofiber defined by $\eta$ :

$$
\eta=\frac{\int_{0}^{2 \pi} \int_{0}^{r_{N F}}(\vec{e} \times \vec{h}) \cdot \vec{z} r d r d \phi}{\int_{0}^{2 \pi} \int_{0}^{\infty}(\vec{e} \times \vec{h}) \cdot \vec{z} r d r d \phi}
$$

We can notice that the fraction of light in the evanescent field for a nanofiber radius of $500 \mathrm{~nm}$ is $23 \%$ and this fraction is higher than $5 \%$ for a radius of $900 \mathrm{~nm}$. As in ref. 
[14], we use this value of $5 \%$ as a criterium to quantify the importance of the evanescent field. It means that for a nanofiber radius of $500 \mathrm{~nm}$ the part of the tapers that are close to the nanofiber can also present a significant evanescent field on a few $\mathrm{mm}$ or $\mathrm{cm}$ (depending on the nanofiber length, as we will see later). This defines a critical length, longer than the nanofiber itself, over which the component is sensitive to dust.
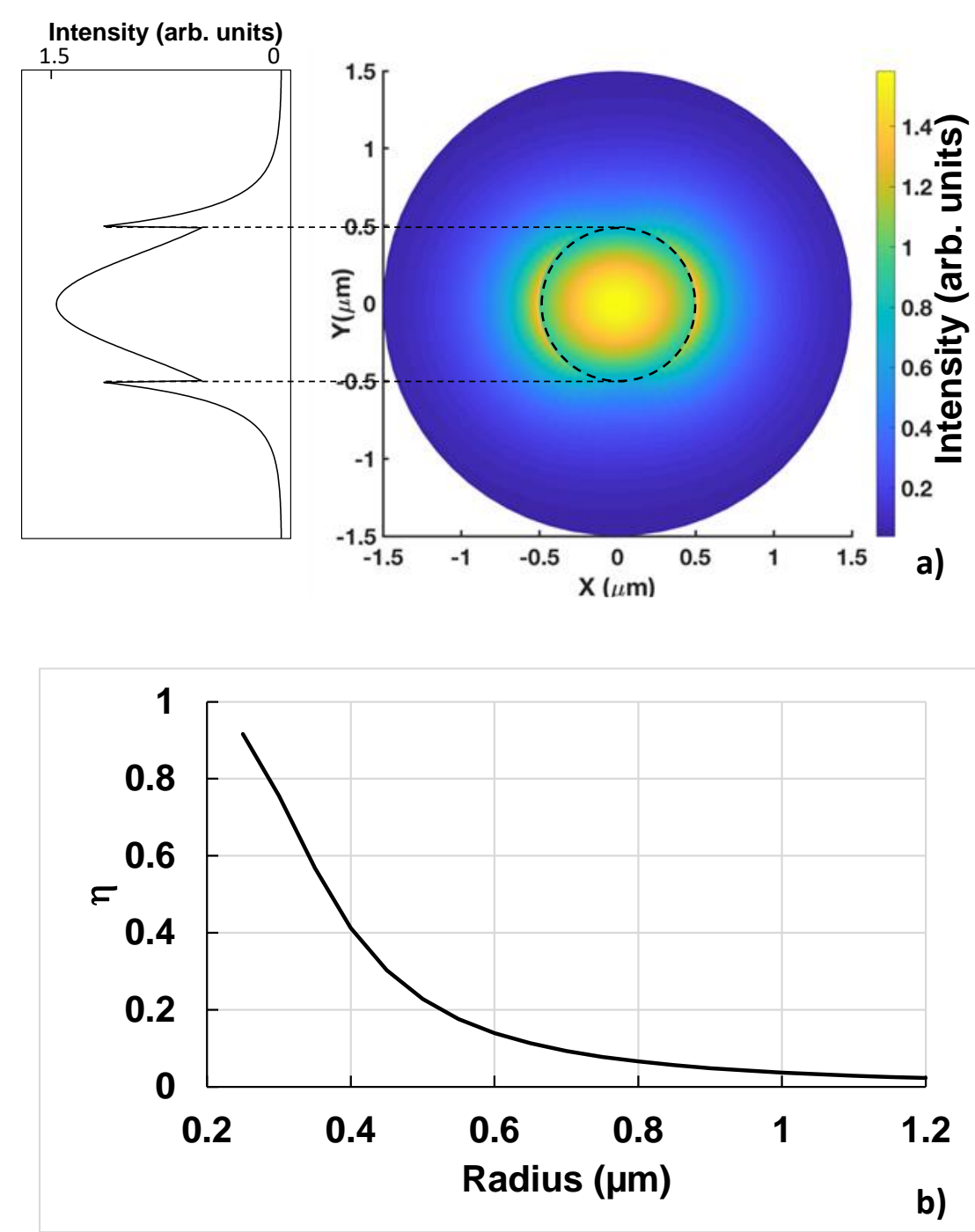

Figure 2. a) Sz, z-component of the Poynting vector of the fundamental mode HE11 at $1.5 \mu \mathrm{m}$ for a nanofiber radius of $500 \mathrm{~nm}$. The top panel is the $\mathrm{Sz}$ cross section in the plane $Y=0$. The dashed line indicates the nanofiber diameter. b) Fraction of the evanescent field $\eta$ versus the radius of the nanofiber at $1.5 \mu \mathrm{m}$. 


\section{Experimental setup and protocol of measurements}

The nanofiber pulling process is described in [18]. We use standard fiber SMF28 for all the experiments. The fiber to be pulled is fusion spliced to two pigtails at both sides before its pulling, with very low losses (typically $0.01 \mathrm{~dB}$ ). A butane flame softens the fiber central part while two computer-controlled translation stages elongate it following the classical "pull and brush" technique. This fabrication process is performed in a class- 5 clean room. Our pulling system enables to fabricate nanofibers with very high optical quality. The optical transmittance of the nanofiber and its tapers is currently around $75-80 \%$ at $1.5 \mu \mathrm{m}$.

Just after fabrication, the nanofiber is fixed from its two untapered parts using a double-side tape piece on a glass holder shown in Figure 3 (bottom left). We pay attention to the tensile strain applied while fixing the nanofiber to keep it stable and straight enough to transmit the maximum of light. The holder is covered by a glass plate, designed to protect the nanofiber without any contact. The large sides of this protection are fully covered with tape. The small sides $\mathrm{A}$ and $\mathrm{B}$ are also covered with tape, but we keep a small aperture to let the access ends of the fiber exit this protection box, denoted as Environment \#1. We can also put this protection box in an airtight box commonly used in clean room to store samples. We keep a small aperture of the plexiglass box to let the fiber ends exit, so that the airtight box is quasi-hermetic. We denote by Environment \#2 the protection box stored in this airtight box. Figure 3 (right) shows Environment \#2. We check that the humidity rates of the two environments are the same $(50 \%)$, as expected. We have also put some silica gel in both environments and check that they evolved similarly during all the experiments we performed. We also put some double-face tape close to the nanofiber in each environment to roughly follow the dusts deposition during the measurements. This preparation stage is realized in the class- 5 clean room.

Figure 3 illustrates the experimental setup we build to perform the long-term measurements of nanofibers transmittance in Environment \#1 and Environment \#2. The setup, entirely fibered, is placed in a standard laboratory, with no special care of the cleanliness but with very little traffic to limit air movement. A fibered laser diode delivering a maximal $\mathrm{cw}$ power of $40 \mathrm{~mW}$ emits a light beam at $1.5 \mu \mathrm{m}$. The beam is split by a $90 / 10$ coupler. The $10 \%$ side is used as a reference and detected by photodiode PD1. The $90 \%$ side is split by a $50 / 50$ coupler, delivering two beams. The first beam is connectorized to the access input fiber of a nanofiber placed in 
Environment \#1 (NF \#1). The second beam is connectorized to the access input fiber of a second nanofiber (NF \#2) placed in Environment \#2.
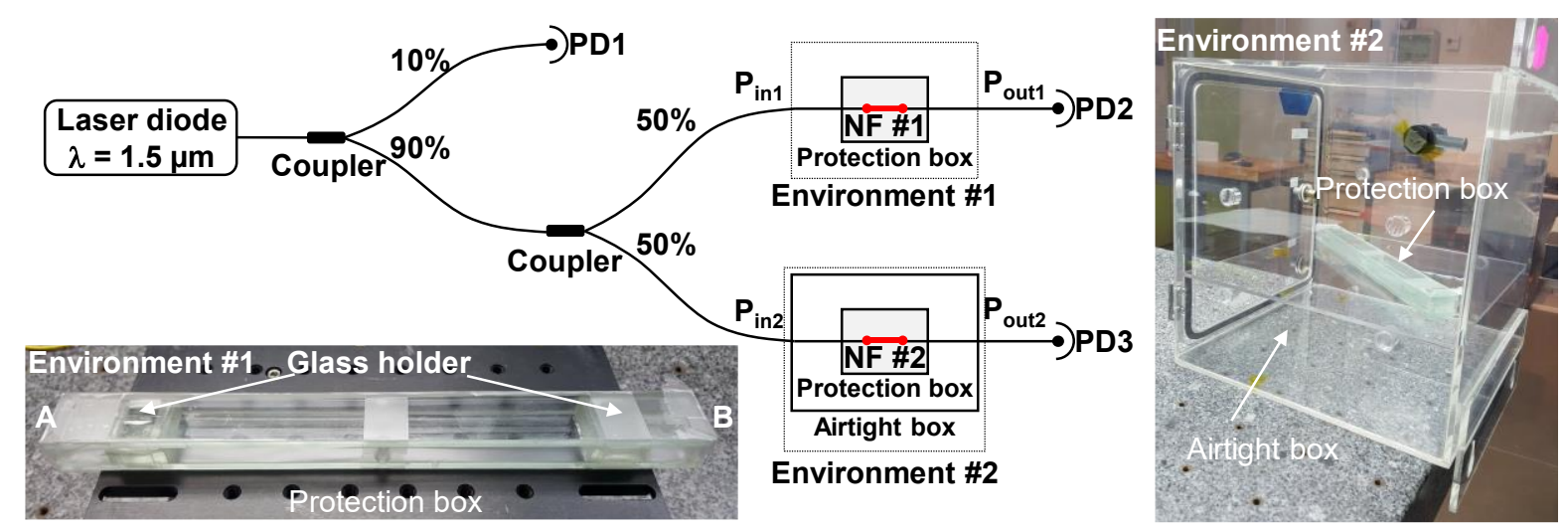

Figure 3. Experimental setup. PD1, PD2, PD3: photodiodes controlled by a computer under a LabVIEW code (ref. Thorlabs PDA 20CS-EC). Bottom left : Environment \#1 (glass holder inside the protection box). A and $B$ : input and output of the box. Right : Environment \#2 (protection box inside the airtight box).

We denote respectively by $T_{1}$ and $T_{2}$ the optical transmittances of NF \#1 and NF \#2. $T_{1,2}$ is defined as the ratio between the output transmitted power $P_{\text {out } 1,2}$ and the input power $P_{\text {in } 1,2}$. The initial transmittances of the nanofibers are measured after their installation in the experimental setup. $\mathrm{P}_{\text {out } 1}$ and $\mathrm{P}_{\text {out2 }}$ are detected by photodiodes PD2 and PD3.

\section{Results and discussion}

3.1 First experiment: comparison of the performances in Environment \#1 and Environment \#2

The first experiment is described hereafter. We draw two nanofibers with the same geometrical parameters: the radius is $500 \mathrm{~nm}$, the nanofiber length is $2 \mathrm{~cm}$ and the total length of the nanofiber with its tapers is $7 \mathrm{~cm}$. The transmittance measurements are divided in two phases: in the first phase (Phase \#1), NF \#1 and NF \#2 are respectively placed in Environment \#1 and Environment \#2. The duration of this Phase \#1 is 85 days. In the second phase (Phase \#2), NF \#1 and NF \#2 are swapped. The duration of this Phase \#2 is 65 days. We continuously record input and output powers, with a sample every minute.

Figure 4 shows the evolution of the optical transmittances versus time. In the insert we firstly observe that the transmittances of both NF \#1 and NF \#2 are not dramatically degraded after 150 days. The transmission loss is only $5 \%$ of the initial 
value in the worst case (i.e., for NF \#1). We also plot linear fits of the optical transmittances, whose slopes give the rate of the optical transmittance decrease in $\% /$ day.

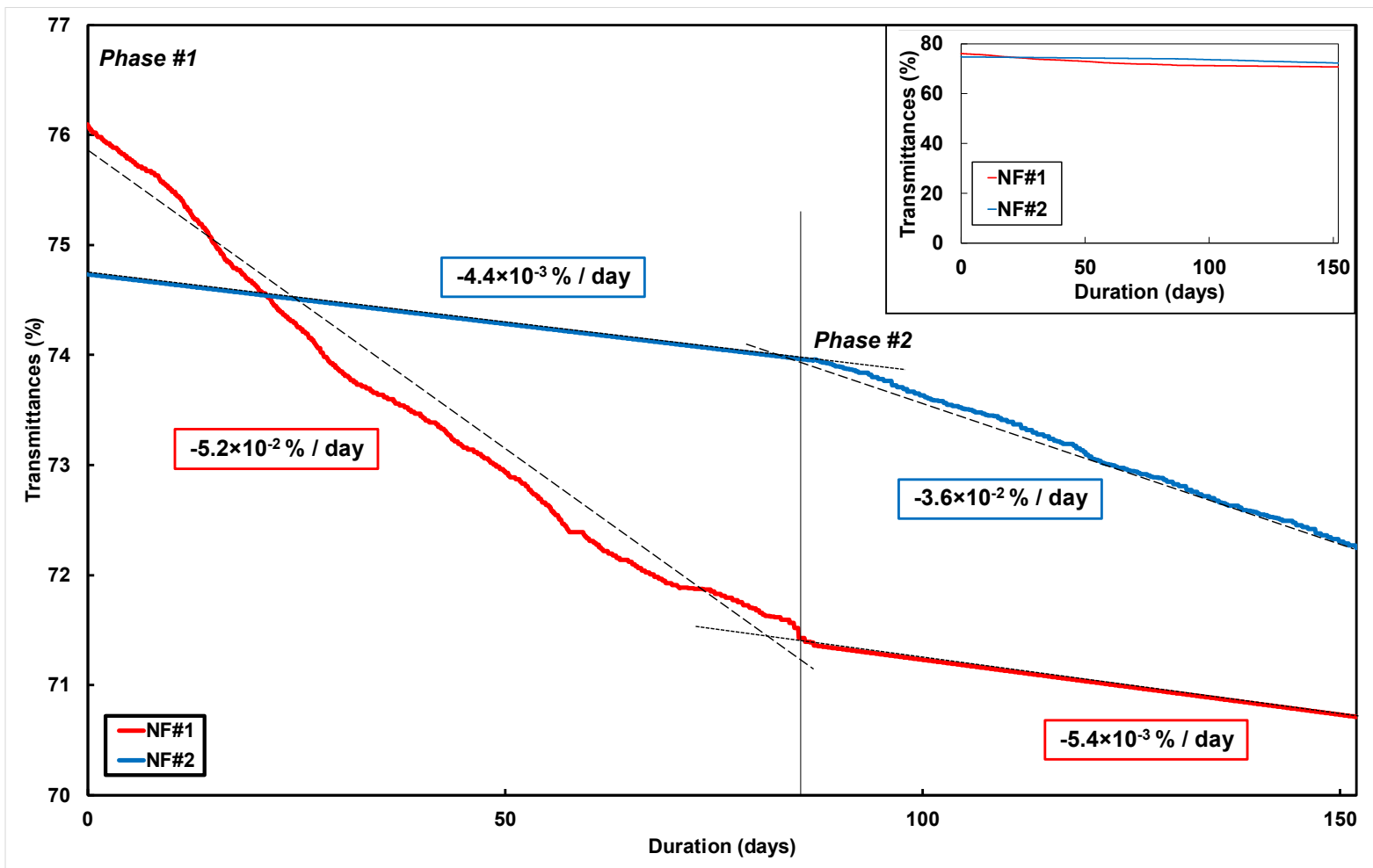

Figure 4. Temporal transmittance measurements at $1.5 \mu \mathrm{m}$ of nanofibers having a radius of $500 \mathrm{~nm}$ and a length of $2 \mathrm{~cm}$. Dashed (resp. dotted) line is for the linear fit of Environment \#1 (resp. \#2) data. The slopes of the linear plots are indicated. Insert : full scale of the plot.

In Environment \#2, the decrease of the transmittance of both nanofibers is slow and regular $\left(-4.4 \times 10^{-3} \% /\right.$ day for NF \#2 and $-5.4 \times 10^{-3} \%$ /day for NF \#1), whereas in Environment \#1 the rate of the decrease is about ten times higher and less regular, but with nevertheless a clear linear tendency $\left(-5.2 \times 10^{-2} \% /\right.$ day for NF \#1 and $3.6 \times 10^{-2} \%$ /day for NF \#2). This linear tendency with no sudden drops of the transmittance shows that no large dust particle has reach the nanofiber during the experiments as this is the case when no particular care is taken when manipulating the nanofiber (experiments with no protection for example, which is not the case here) and that the dust particles involved in the decrease of the transmittances are of nanometric or at the most sub-micrometric sizes.

It is interesting to note that the behaviors of the two nanofibers are symmetrical, which explains the origin of the dusts that degrade the transmittances. Indeed, if these dusts were initially present in the protection box, the decrease of the 
transmittance of NF \#1 would have continue with the same behavior when put in Environment \#2, which is not the case. This clearly shows that the dusts that degrade the nanofibers transmittance are brought by the weak but permanent exchange that exists between the external atmosphere which is continuously renewed and the protection box through its small apertures ( $A$ and $B$ on Figure 3 ). As a confirmation of this explanation, we note that there are much more visible dust particles accumulated on the double-side tape placed in Environment \#1 at the end of the measurements than on the tape put in Environment \#2.

Other inner causes such as carbon particles deposition during the fabrication or micro-cracks at the surface of the nanofiber can also lead to a low decrease of the transmission. This experiment shows that in Environment \#1 their contribution is negligible compared to the contribution of the dusts coming from outside. Their contribution becomes more visible in Environment \#2, where the degradation cause may be a conjugated effect of both inner causes and dusts.

\subsection{Second experiment: repeatability of the results}

In a second experiment, to check to repeatability of our results, we made three measurements on several tens of days in the two environments, changing each time the nanofibers (but keeping the same geometrical parameters). In Figure 5, we represent the different values of the linear fit slopes for each test. The mean and the standard deviation of these linear fit slopes are also calculated. These figures enable to predict the value of the transmittance of the nanofibers on very long durations: for example, according to these fits, the transmittance in Environment \#1 would decrease by $10 \%$ after 6 months from its initial value. In Environment \#2, it would decrease by $10 \%$ from its initial value after more than 4 years. The difference of the slopes for a given environment is mainly attributed to the differences of the components inherent to the fabrication process. In this range of radii, the uncertainty and the uniformity on the nanofiber diameters are very good (respectively estimated to be $+/-15 \mathrm{~nm}$ and below $10 \%$ ), nevertheless leading to a small variation of the fraction of the evanescent field hence to a variation of the sensitivity of the field to the dusts or surface defaults. 

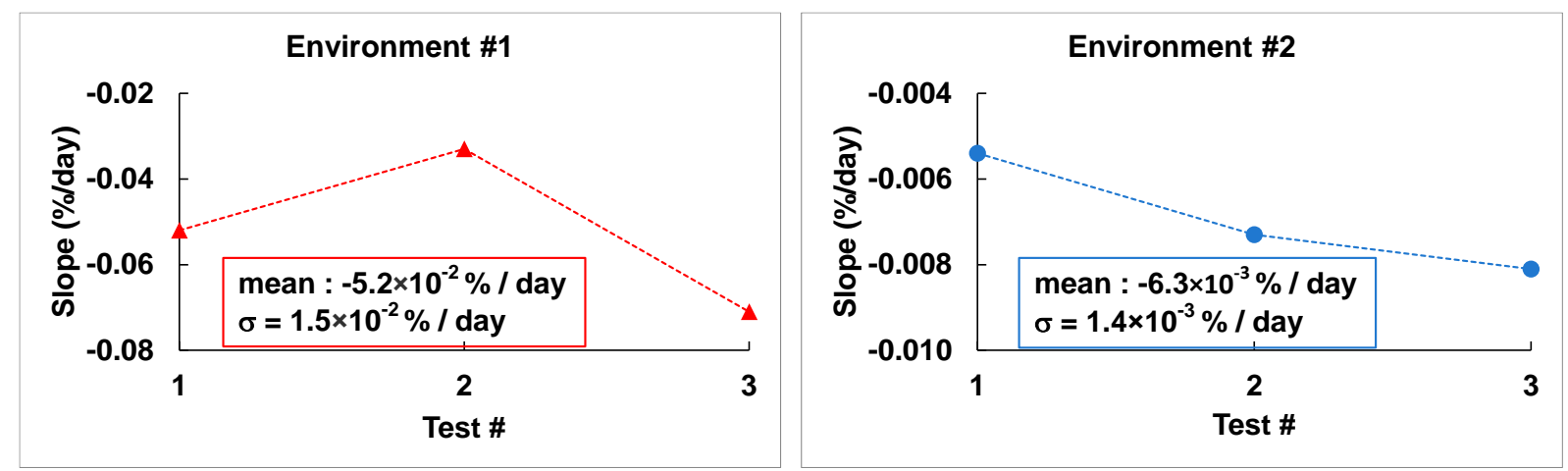

Figure 5. Linear fit slope values of three tests in Environment \#1 and in Environment \#2. The nanofiber radii are $500 \mathrm{~nm}$, their lengths are $2 \mathrm{~cm}$. The dashed lines are just a guide for the eyes. Insert : mean and standard deviation values.

\subsection{Third experiment: influence of the nanofiber length}

In a third experiment, we study the influence of the nanofiber length on the degradation of the transmittance. We fabricate two nanofibers NF \#1 and NF \#2 with a radius of $500 \mathrm{~nm}$ and a length of $7 \mathrm{~cm}$. The total length of the nanofiber plus its tapers is $20 \mathrm{~cm}$.

To compare with the previous results obtained with the $2 \mathrm{~cm}$ long nanofiber, we have plotted the profile of our tapers for the two nanofiber lengths in Figure 6 . Over $0.7 \mathrm{~cm}$ for the $2 \mathrm{~cm}$ long nanofibers and over $2.6 \mathrm{~cm}$ for the $7 \mathrm{~cm}$ long nanofibers (indicated by the grey areas on Figure 6), the radius of the taper remains smaller than $900 \mathrm{~nm}$, meaning that the fraction of light in the evanescent field is higher than $5 \%$. So, for the $2 \mathrm{~cm}$ long nanofiber, the critical length where the optical field is sensitive to dust is $3.4 \mathrm{~cm}$. For the $7 \mathrm{~cm}$ long nanofiber, this critical length is $12.2 \mathrm{~cm}$. 


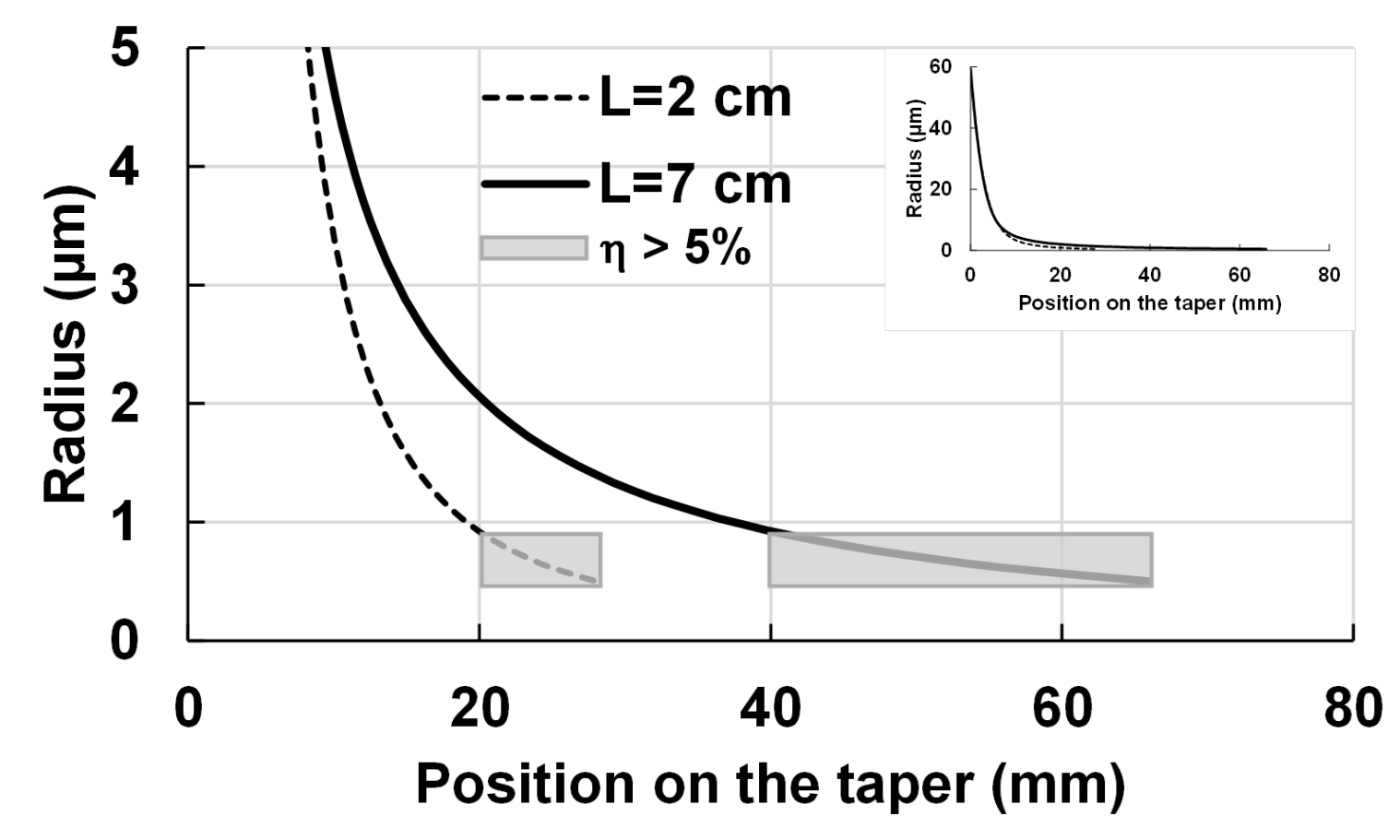

Figure 6. Zoom on the taper profiles of the $2 \mathrm{~cm}$ long nanofiber (dashed line) and of the $7 \mathrm{~cm}$ long nanofiber (full line). The radius of the nanofibers is $500 \mathrm{~nm}$. Grey boxes indicate the critical length, i.e. the portion of the taper where the fraction of the evanescent field is higher than $5 \%$. Insert : full taper profiles.

NF \#1 is placed in Environment \#1 and NF \#2 in Environment \#2. We perform the transmittance measurement over 45 days. The results are shown on Figure 7. Compared to the results of Figure 5, we observe that the length of the nanofiber plus its tapers has no significant impact on the degradation rate $\left(-5.3 \times 10^{-3} \% /\right.$ day for Environment \#1 and $-6.9 \times 10^{-2} \%$ day for Environment \#2) and that putting the nanofiber in Environment \#2 still slows down this slope by a factor of almost ten, as this was the case for the $2 \mathrm{~cm}$ long nanofibers. 




Figure 7. Temporal transmittance measurements at $1.5 \mu \mathrm{m}$ of nanofibers having a radius of $500 \mathrm{~nm}$ and a length of $7 \mathrm{~cm}$. Dashed (resp. dotted) line is for the linear fit of Environment \#1 (resp. \#2) data. The slopes of the linear plots are indicated.

\section{Conclusion}

In this paper, we present long-term and repeatable measurements of nanofiber optical transmittances during several months, performed in two different environments with two different nanofiber waist lengths and a radius of $500 \mathrm{~nm}$. At this radius, the evanescent field is important, and the nanofibers are then very sensitive to dust deposition on the surface which is a strong issue for efficient storage and long-term experiments. We show that a simple protection that can be easily fabricated in any laboratory is already highly efficient to store nanofibers on very long period (months and even years) without any significant reduction of its optical transmittance. Moreover, an additional degree of cleanliness provided by an airtight box enables to further slowdown the degradation rate by a factor of ten. The experiments were conducted three times and the results were very repeatable. The use of complex solutions such as embedding the nanofiber in a polymer is then not useful to protect a nanofiber. We also demonstrate that the length of the nanofiber and its tapers has no significant impact on the transmittance degradation, so several 
$\mathrm{cm}$ length nanofibers that are typically used for nonlinear experiments $[5,8]$ can be stored as efficiently as shorter ones often used in other applications, such as quantum optics.

Funding: This work is supported by the French National Research Agency (ANR) (FUNFILM-ANR-16-CE24-0010-03).

\section{References}

[1] J. E. Hoffman, S. Ravets, J. A. Grover, P. Solano, P. R. Kordell, J. D. WongCampos, L. A. Orozco, S. L. Rolston, "Ultrahigh transmission optical nanofibers", AIP advances, 4 (6) p. 068124 (2017).

[2] M. Joos, C. Ding, V. Loo, G. Blanquer, E. Giacobino, A. Bramati, V. Krachmalnicoff, and Q. Glorieux, "Polarization control of linear dipole radiation using an optical nanofiber", Physical Review Applied, 9(6), 064035 (2018).

[3] A. Maimaiti, V. G. Truong, M. Sergides, I. Gusachenko, and S. Nic Chormaic, "Higher order microfibre modes for dielectric particle trapping and propulsion", Sci. Rep. 5, 9077 (2015).

[4] L. Tong, "Micro/nanofibre optical sensors: challenges and prospects", Sensors 18.3903 (2018).

[5] M. Bouhadida, J. -C. Beugnot, P. Delaye, K. P. Huy, S. Lebrun, (2019), "Highly efficient and reproducible evanescent Raman converters based on a silica nanofiber immersed in a liquid", Applied Physics B, 125(12), pp. 1-7 (2019).

[6] J.-C. Beugnot, S. Lebrun, G. Pauliat, H. Maillotte, V. Laude, and T. Sylvestre, "Brillouin light scattering from surface acoustic waves in a subwavelength-diameter optical fibre", Nat. Commun. 5, 5242 (2014).

[7] G. Fanjoux, J. Chrétien, A. Godet, K. Phan-Huy, J. -C. Beugnot, T. Sylvestre, "Demonstration of the evanescent Kerr effect in optical nanofibers", Optics express, 27(20), pp. 29460-29470 (2019).

[18] Ph. Delaye, T. Liu, E. Mer, M. Bouhadida, and S. Lebrun " CW generation of photon pairs in silica nanofibers using single- and multi-longitudinal mode pumps", submitted to Phys. Rev. A.

[9] T. Yoshie, L. Tang, and S.-Y. Su, "Optical microcavity: sensing down to single molecules and atoms", Sensors 11, pp. 1972-1991 (2011). 
[10] E. Vetsch, S. T. Dawkins, R. Mitsch, D. Reitz, P. Schneeweiss, and A. Rauschenbeutel, "Nano_berbased optical trapping of cold neutral atoms", IEEE J. Sel. Top. Quantum Electron. 18, pp. 1763-1771 (2012).

[11] R. Kumar, V. Gokhroo, and S. Nic Chormaic, "Multilevel cascaded electromagnetically induced transparency in cold atoms using an optical nanofibre interface", New J. Phys. 17, 123012 (2015).

[12] P. Solano, J. A. Grover, J. E. Hoffman, S. Ravets, F. K. Fatemi, L. A. Orozco, and S. L. Rolston, "Optical nanofibers: a new platform for quantum optics", Advances In Atomic, Molecular, and Optical Physics, 66, pp. 439-505 (2017).

[13] T. A. Birks, W. J. Wadsworth, and P. St J. Russell. "Supercontinuum generation in tapered fibers", Optics letters 25(19) pp. 1415-1417 (2000).

[14] M. Fujiwara, K. Toubaru, S. Takeuchi, "Optical transmittance degradation in tapered fibers", Optics Express, 19 (9), pp. 8596-8601 (2011).

[15] M. Gregor, A. Kuhlicke, and O. Benson, "Soft-landing and optical characterization of a preselected single fluorescent particle on a tapered optical fiber", Opt. Express 17, pp. 24234-24243 (2009).

[16] F. Xu and G. Brambilla, "Preservation of micro-optical fibers by embedding", Jpn. J. Appl. Phys. 47, pp. 6675-6677 (2008).

[17] A.W. Snyder, J. Love, "Optical Waveguide Theory", Chapt. 14, Kluwer Academic Publisher, Boston/Dortrecht/London (2000).

[18] L. Shan, G. Pauliat, G. Vienne, L. Tong, L., and S. Lebrun "Stimulated Raman scattering in the evanescent field of liquid immersed tapered nanofibers", Applied Physics Letters, 102(20), p. 201110 (2013). 